Check for updates

Cite this: RSC Adv., 2019, 9, 4563

\title{
Enhanced thermal conductance at the graphene- water interface based on functionalized alkane chains
}

\author{
Shuyu Chen, (D) $\dagger^{\mathrm{ab}}$ Ming Yang, $\dagger^{\mathrm{a}}$ Bin Liu, ${ }^{\mathrm{a}}$ Min Xu, ${ }^{a}$ Teng Zhang, ${ }^{\text {*c }}$ Bilin Zhuang, ${ }^{d}$ \\ Ding Ding, ${ }^{\text {*e }}$ Xiulan Huai ${ }^{\mathrm{ab}}$ and Hang Zhang (iD) *ab
}

Highly efficient thermal transport between graphene and water is crucial in applications such as microscopic heat dissipation, solar steam generation, sea-water desalination, and thermally conductive composites. However, a practical approach for enhancing thermal transport across graphene-water interfaces is lacking. We propose an effective and universal method to improve thermal-transport properties at the interface between multilayer graphene and water by a factor of $\sim 4$ by grafting functionalized groups onto graphene. The most improved interfacial thermal conductance was $121.0 \pm$ 11.4 $\mathrm{MW} \mathrm{m}^{-2} \mathrm{~K}^{-1}$. This design is compatible with industrial processes. We also undertook molecular-level analyses to unveil the underlying mechanism for heat-transport enhancement. This study could provide new approaches for engineering heat transport across two-dimensional materials and water interfaces.

Received 1st December 2018

Accepted 24th January 2019

DOI: $10.1039 / \mathrm{c} 8 \mathrm{ra0} 0879 \mathrm{~d}$

rsc.li/rsc-advances

graphene and water by molecular dynamics (MD) simulations.

\section{Introduction}

Graphene, ${ }^{1}$ as a material with excellent mechanical properties, ${ }^{2}$ ultrahigh electric and thermal conductivities, ${ }^{3-5}$ and special optical properties, ${ }^{6}$ has garnered attention worldwide. Among these features, its excellent thermal properties have great potential for thermal management in fields such as electronics and biochemistry. ${ }^{7-9}$ If the characteristic dimensions of objects reach nanometer scales, thermal resistances on the interfaces have increasingly important roles and even dominate thermaltransport properties..$^{10-12}$

Some studies have demonstrated that the thermal conductivity of graphene can reach approximately $5000 \mathrm{~W} \mathrm{~m}^{-1} \mathrm{~K}^{-1}$ at room temperature, ${ }^{3,13}$ but the interfaces between graphene and soft materials demonstrate only mediocre interfacial thermal conductance. $^{14,15}$ Therefore, attention has focused on improving thermal-transport properties across graphene-soft-material interfaces. Ma et al. ${ }^{16}$ suggested that charge decoration on graphene can decrease the Kapitza resistance between graphene and water. Alexeev et al. ${ }^{17}$ found that the number of the layers in fewlayer graphene determined the Kapitza resistance between

anstitute of Engineering Thermophysics, Chinese Academy of Sciences, Beijing 100190, China. E-mail: zhanghang@iet.cn

${ }^{b}$ University of Chinese Academy of Sciences, Beijing 100049, China

'Materials Science, Schrödinger, New York, NY10035, USA. E-mail: zhteg4@gmail.com ${ }^{d}$ Institute of High Performance Computing, A*STAR Research Entities, Singapore 138632

${ }^{e}$ Institute of Materials Research and Engineering, $A^{*}$ STAR Research Entities, Singapore 138634.E-mail:ding_ding@imre.a-star.edu.sg

$\dagger$ Shuyu Chen and Ming Yang contributed equally to this work.
Cao et al.$^{18}$ found that the interfacial thermal resistance between graphene and water could be reduced with interlayer functionalization by oxygen atoms. Wang et al. ${ }^{19}$ demonstrated that intercalated water can enhance thermal transport between the cell membrane-graphene interface.

Functionalization on graphene is considered an effective approach to improve thermal transport across interfaces between graphene and soft materials. ${ }^{20,21}$ Yang et al. ${ }^{22}$ improved the solar-to-vapor conversion efficiency by functionalizing graphene with hydrophilic groups through experiments. Teng et al. ${ }^{23}$ improved the thermal conductivity of GNS-filled epoxy composites through non-covalent functionalization to graphene in experiments. Wang et al. ${ }^{24}$ used MD simulation to study the interfacial thermal resistance in polymer composites reinforced by various covalently functionalized types of graphene. Wang et al. ${ }^{25}$ investigated thermal transport across graphene-polymer interfaces functionalized with end-grafted polymer chains using MD simulations. Tang et $a .^{26}$ found thermal transport between graphene and polymers to be enhanced with alkane chains functionalized to graphene via MD simulations. Lin et al. ${ }^{27}$ found that alkyl-pyrene as a linker candidate could enhance out-of-plane thermal conductance of the graphene-organic compound interface through MD simulations. However, very few studies have utilized functionalized graphene (FG) to achieve highly efficient thermal transport across graphenewater interfaces.

We report a theoretical study on improving the thermaltransport properties of graphene-water interfaces by grafting alkane chains $\left(\mathrm{C}_{n} \mathrm{H}_{2 n+1}\right)$ on graphene. To provide a systematic 


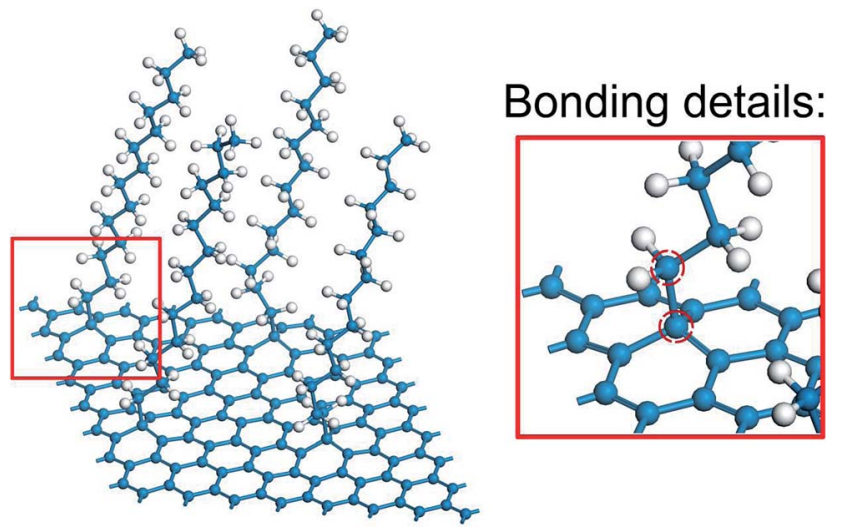

Fig. 1 Functionalized graphene (FG) structure by alkane chains and bonding details (schematic). Blue spheres represent carbon atoms and white spheres represent hydrogen atoms. In the FG models, the alkane chains $\left(\mathrm{C}_{n} \mathrm{H}_{2 n+1}\right)$ were grafted in an orderly fashion to one side of the graphene surface by $\mathrm{sp}^{3}$-hybridized $\mathrm{C}-\mathrm{C}$ bonds.

study, a series of FG-water models were built with different alkane chain lengths and surface coverage for tunable interfacial thermal conductance. This work could provide new insights for applications such as self-assembled monolayers, ${ }^{28}$ nanoscale microchannels, ${ }^{29}$ microelectronics, ${ }^{30}$ nanofluids, ${ }^{31}$ solar thermal steam generation ${ }^{22}$ and biological cells. ${ }^{19}$

\section{Methods}

In the view of the practical applications and literature mentioned above, ${ }^{16-18}$ multilayer graphene (MLG) was constructed and simulated in the present study, and the outermost graphene was functionalized. In the FG models, the alkane chains $\left(\mathrm{C}_{n} \mathrm{H}_{2 n+1}\right)$ were grafted in an orderly manner to one side of the graphene surface by $\mathrm{sp}^{3}$-hybridized $\mathrm{C}-\mathrm{C}$ bonds ${ }^{32}$ (Fig. 1). Then, the water molecules were fully filled into the remaining spaces of the simulation boxes with density of $1 \mathrm{~g} \mathrm{~cm}^{-3}$. All the models were designed in a box of $20 \AA \times 20 \AA \times 300 \AA$, with periodic boundary conditions applied in all directions. Thereafter, the structures were relaxed with the isothermal-isobaric (NPT) ensemble, and optimized by the conjugate gradient method.

The interfacial thermal conductance was calculated through non-equilibrium molecular dynamics (NEMDs), which are based on the Muller-Plathe approach..$^{33}$ In NEMDs, heat sink and heat source were applied. The heat flux and temperature field were created by exchanging the momentum between atoms in heat sink and heat source. Periodic boundary conditions were applied in all directions. ${ }^{34}$ Some details are shown in Fig. 2(a). All NEMD simulation processes were carried out in Forcite. ${ }^{35}$ DREIDING, a generic force field, was used to describe the interatomic interactions in the system. DREIDING has been used widely to simulate the structures and dynamics of organic and main-group inorganic

(a)

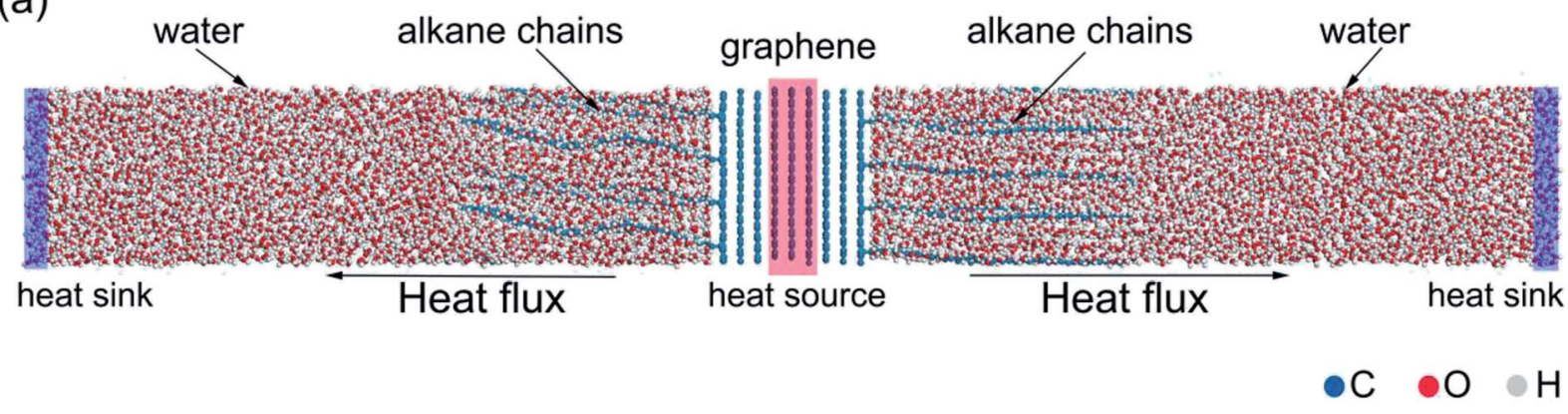

(b)

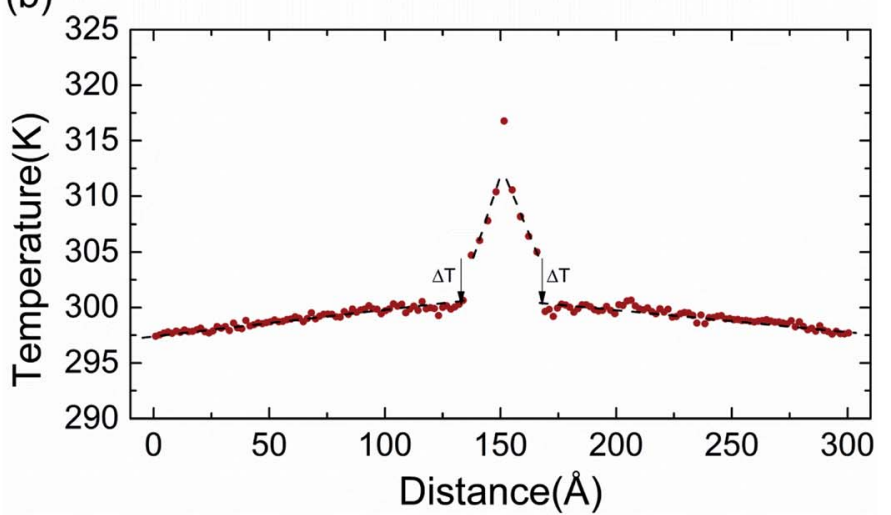

Fig. 2 (a) A functionalized graphene-water interface in NEMD simulation (schematic). In NEMD, heat sink and heat source were applied. The heat flux and temperature field were created by exchanging the momentum between atoms in the heat sink and heat source. (b) Temperature profile of a functionalized graphene-water interface. The temperature changes linearly along the heat-flux direction in bulk water and has a sharp drop at the interface. 
molecules, and the van der Waals interactions are described by the Lennard-Jones potential. ${ }^{36}$ DREIDING is suitable for graphene-water systems.

A small time step of 0.5 fs was chosen for all simulations. Prior to the simulations, all models were relaxed using an isothermal-isobaric (NPT) ensemble at $300 \mathrm{~K}$ and $1 \mathrm{~atm}$ for 1 ns to release residual stresses. To further relax the structures, a constant volume in a canonical (NVT) ensemble at $300 \mathrm{~K}$ was used. Then, the systems were simulated using a microcanonical (NVE) ensemble with two thermostated regions. The momentum exchange between the heat sink and heat source was done every 1000 time steps. After the simulations had reached a steady state, the temperature profiles were averaged over $4 \mathrm{~ns}$ to calculate the interfacial thermal conductance between graphene and water.

The interfacial thermal conductance $(G)$ was calculated as

$$
G=q / \Delta T
$$
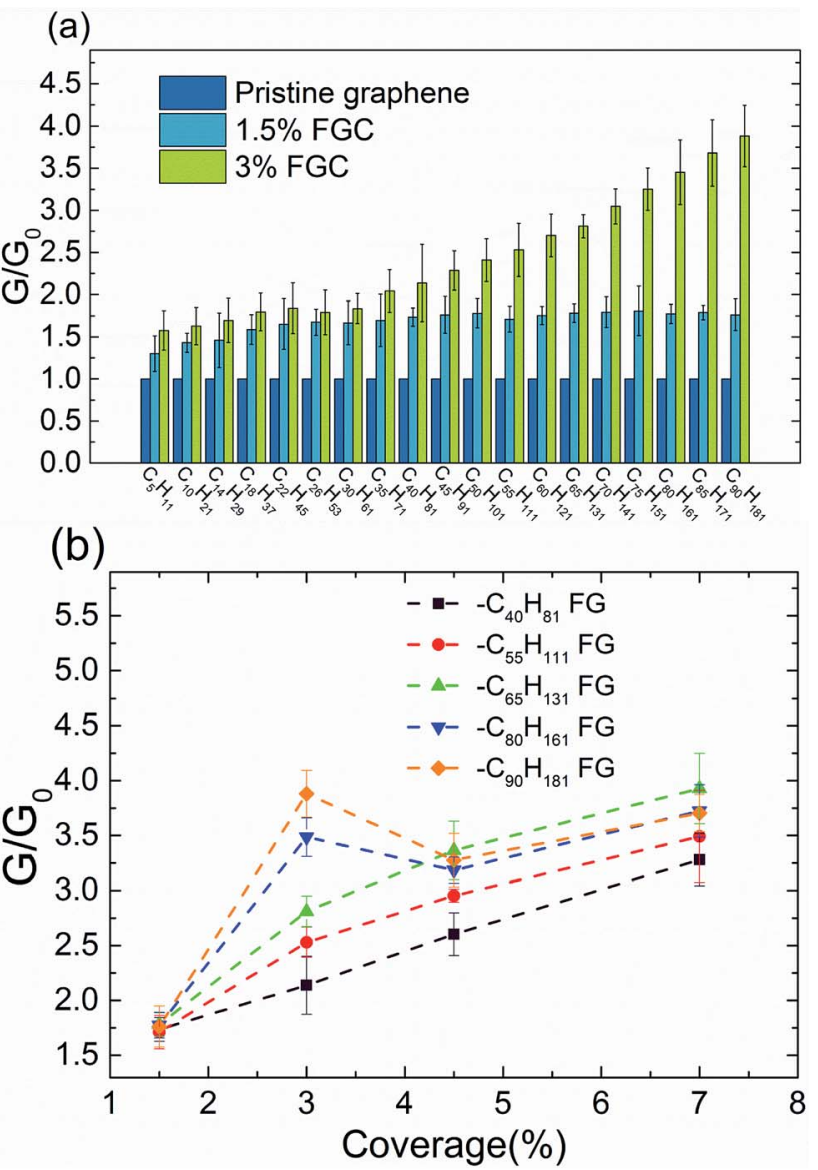

Fig. 3 Relative interfacial thermal conductance $G / G_{0}$ of water and functionalized graphene compared with a pristine graphene-water interface. (a) Different length of alkane chains. (b) Different functionalgroup coverage (FGC). The interfacial thermal conductance was improved significantly by surface functionalization. The improvement increased with the FGC in general, except when FGC was $>3 \%$ in long chains (over $-\mathrm{C}_{75} \mathrm{H}_{151} \mathrm{FG}$ ). where $\Delta T$ is the temperature drop at the interface between graphene and water, and $q$ is the heat flux through the interface. $q$ was calculated as

$$
q=\frac{1}{2 A} \frac{\mathrm{d} \Delta E(t)}{\mathrm{d} t}
$$

where $\Delta E$ is the energy variation in the thermostat. In our simulations, we used a constant energy exchange rate, and approximated heat fluxes were achieved across all models.

\section{Results and discussions}

First, we simulated the interfacial thermal conduction between pristine graphene and water. The temperature profile was found to change linearly along the heat-flux direction in bulk water and had a sharp drop at the graphene-water interface (Fig. 2(b)). The interfacial thermal conductance $(G)$ was calculated by heat flux over the temperature drop. Then, the interfacial thermal conductance of FG with different alkane chains $\left(-\mathrm{C}_{n} \mathrm{H}_{2 n+1}\right)$ was calculated. To have a direct insight of the effect of functionalization of the graphene surface on the interface, the relative interfacial thermal conductance $G / G_{0}$ (where $G_{0}$ is the interfacial thermal conductance between pristine graphene and water) was carried out to describe the enhancement. Here, the $G_{0}$ between five-layer graphene and water was calculated to be $30.8 \pm 2.2 \mathrm{MW} \mathrm{m}{ }^{-2} \mathrm{~K}^{-1}$, which is in accordance with the reported thermal resistance of graphene-water: $\sim 4.0 \times 10^{-8} \mathrm{~K}$ $\mathrm{m}^{2} \mathrm{~W}^{-1}\left(\sim 25.0 \mathrm{MW} \mathrm{m}{ }^{-2} \mathrm{~K}^{-1}\right)$ from Alexeev et al. ${ }^{17} \sim 3.2 \times 10^{-8}$ $\mathrm{K} \mathrm{m}^{2} \mathrm{~W}^{-1}\left(\sim 31.2 \mathrm{MW} \mathrm{m^{-2 }} \mathrm{K}^{-1}\right)$ from Ma et al. ${ }^{16}$ and $\sim 2.9 \times$ $10^{-8} \mathrm{~K} \mathrm{~m}^{2} \mathrm{~W}^{-1}\left(\sim 34.5 \mathrm{MW} \mathrm{m}{ }^{-2} \mathrm{~K}^{-1}\right)$ from Cao et al. ${ }^{18}$ The functional group coverage (FGC) was defined as the ratio of the number of alkane chains to the carbon atoms of the outermost layer of graphene.

The interfacial thermal conductance was found to be improved significantly by surface functionalization. The best improved interfacial thermal conductance reached $121.0 \pm 11.4$

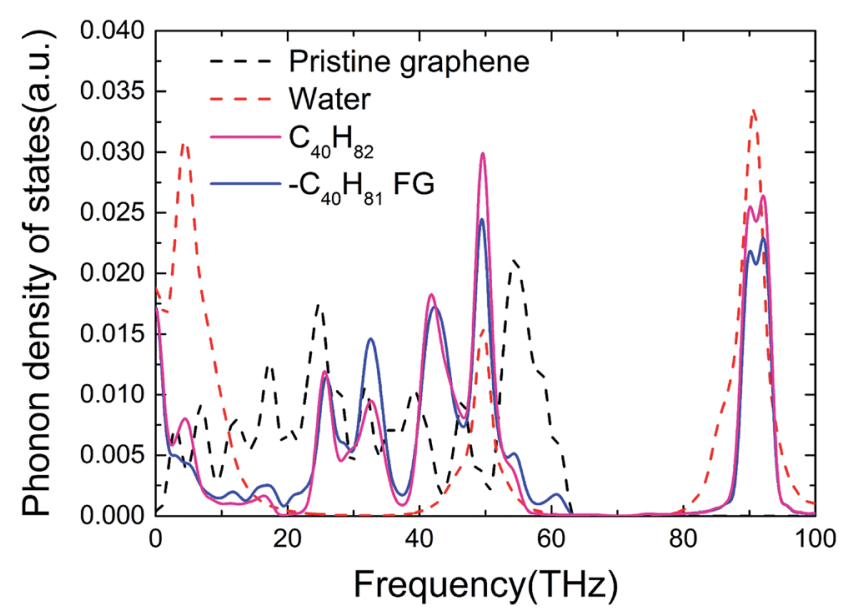

Fig. 4 Phonon DOSs of pristine graphene, water, $-\mathrm{C}_{40} \mathrm{H}_{81}$ functionalized graphene and the corresponding functional group in stable state $\left(\mathrm{C}_{40} \mathrm{H}_{82}\right)$. The functionalized alkane alters the phonon vibration modes of graphene dramatically. 

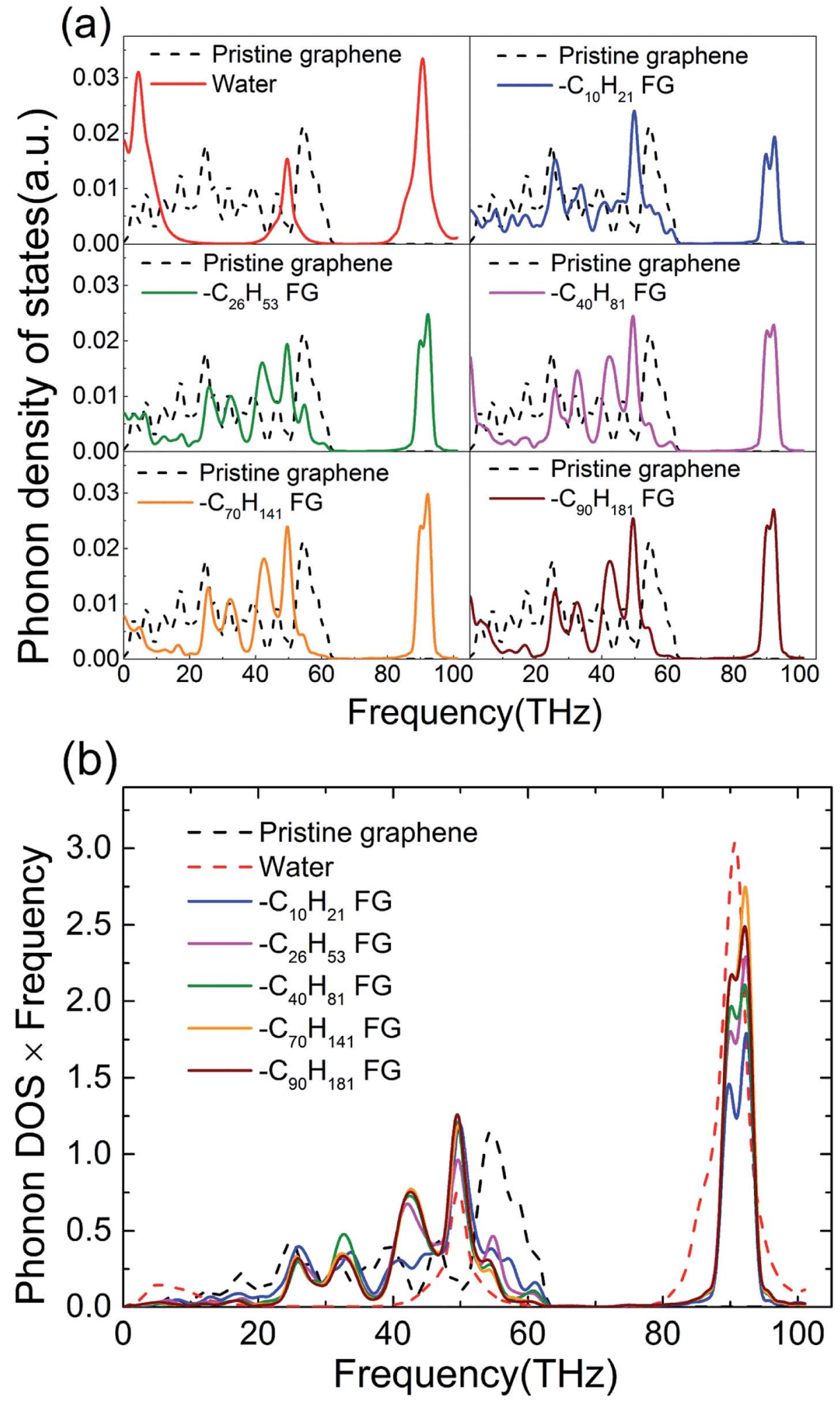

Fig. 5 (a) Phonon DOSs and (b) phonon DOSs multiplied by the frequency of functionalized graphene with various numbers of $C$ atoms in alkane chains compared with water and pristine graphene. The distribution of energy is indicated by the phonon DOSs multiplied by frequency.

MW $\mathrm{m}^{-2} \mathrm{~K}^{-1}$ at $-\mathrm{C}_{65} \mathrm{H}_{131}$ FG in $7 \%$ FGC. The interfacial thermal conductance increased by a factor of 1.3-1.8 under a FGC of $1.5 \%$. At $3 \%$ FGC, the improvement could be as much as fourfold. At 3\% FGC, the interfacial thermal conductance was found to increase as the alkane chains became longer. Such a trend was found at $1.5 \%$ FGC for some short chains, but the increasing trend was not obvious if the increase was more than $-\mathrm{C}_{18} \mathrm{H}_{37}$. To carry out a systematic investigation, FGs were 
simulated further with various chain lengths $\left(-\mathrm{C}_{40} \mathrm{H}_{81}\right.$, $-\mathrm{C}_{55} \mathrm{H}_{111},-\mathrm{C}_{65} \mathrm{H}_{131},-\mathrm{C}_{80} \mathrm{H}_{161}$ and $\left.-\mathrm{C}_{90} \mathrm{H}_{181}\right)$ and different FGCs $(1.5 \%, 3 \%, 4.5 \%$ and $7 \%)$. In general, the relative interfacial thermal conductance increased with coverage (Fig. 3(b)), and showed a clear trend with respect to the alkane length at $3 \%$ FGC. However, the relative interfacial thermal conductance did not increase if the coverage was $>3 \%$ in $-\mathrm{C}_{80} \mathrm{H}_{161} \quad \mathrm{FG}$ and $-\mathrm{C}_{90} \mathrm{H}_{181}$ FG.

To reveal the underlying mechanism behind enhancement of the thermal conductance of the graphene-water interface, we investigated the atomic and corresponding phononic information of the system. Hydrogen atoms in the alkane chains had an important role in this enhancement because they provide extra energy-transfer paths between carbon atoms in graphene and the atoms in water. Studies have shown that hydrogen bonds can improve the efficiency of interfacial thermal transport significantly if stronger hydrogen bonds "pull" the organic molecules closer to the interface and establish better contact. ${ }^{\mathbf{1 4}}$ Greater similarity in phonon vibration properties has been reported to facilitate interfacial heat exchange. ${ }^{11,37-43}$ Furthermore, the spectral information of phonons was calculated (Fig. 4). The phonon density of states (PDOS) were calculated by taking the fast Fourier transform of atomistic velocity autocorrelation functions (VAFs). ${ }^{27,44}$ Specifically, the density of states, $\omega$, was given by

$$
D(\omega)=\int_{0}^{\tau}\langle\nu(t) \nu(0)\rangle \exp (-i \omega t) \mathrm{d} t
$$

where $\nu(0)$ and $\nu(t)$ are the velocities of each atom at initial time and at time $t$, respectively.

Fig. 4 shows the PDOS spectra of pristine graphene, water, a typical FG and its functional groups. It can be clearly observed that the functionalized alkane dramatically altered the graphene phonon vibration modes of graphene. Water molecules are rich in $\mathrm{H}-\mathrm{O}$ bonds with a vibration frequency as high as $\sim 3000 \mathrm{~cm}^{-1}(\sim 90 \mathrm{THz}),{ }^{45}$ which is in accordance with the peaks at $90 \mathrm{THz}$ in Fig. 4. Graphene is formed by in-plane carbon atoms combined by $\mathrm{sp}^{2}$-hybridized $\mathrm{C}-\mathrm{C}$ bonds, which cannot provide ultra-high vibration frequencies. Functionalization of alkane chains is based on methyl and methylene groups, in which the $\mathrm{sp}^{3}$-hybridized $\mathrm{C}-\mathrm{C}$ bonds and $\mathrm{C}-\mathrm{H}$ bonds also exhibit high vibration frequencies at $\sim 3000 \mathrm{~cm}^{-1}$. $^{45}$ Thus, we can infer that the two high-frequency peaks from FG corresponded mainly to $\mathrm{C}-\mathrm{H}$ bonds in the functional groups (Fig. 4). In addition, the alkane-functionalization itself had vibration modes with frequency $<5 \mathrm{THz}$, and distortion of the graphene plane due to functionalization also impacts low frequency phonons. These low-frequency phonons due to functionalization provided better overlap with those in amorphous water. In addition, a larger overlap area $\sim 50 \mathrm{THz}$ and $\sim 90 \mathrm{THz}$ between the PDOS spectra of water and FG due to the similar $\mathrm{sp}^{3}$ hybridized carbon served as another factor for enhancement of thermal conductance.

PDOSs and PDOSs multiplied by the vibration frequency were calculated for graphene functionalized with alkanes of various chain lengths to examine further the vibration overlap between water and FGs (Fig. 5). The energy distribution can be described by the product of phonon DOSs and frequency (Fig. 5(b)), so a large fraction of energy was carried by highfrequency heat carriers in FG. Though heat carriers of high frequency do not contribute as much to in-plane thermal transport as diffusion, ${ }^{37,39,46}$ they are important in thermal transport across interfaces. ${ }^{\mathbf{1 1}}$ As demonstrated by the significant enhancements in interfacial thermal conductance between FG and water, heat carriers of high frequencies in FG served as critical energy carriers beyond the low-frequency propagon.

Because longer alkane chains and higher coverage provide more high-frequency heat carriers that could enhance (a) $-\mathrm{C}_{40} \mathrm{H}_{81}$ FG of $1.5 \%$ FGC

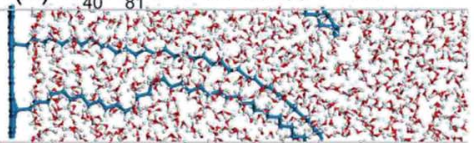

(b) $-\mathrm{C}_{40} \mathrm{H}_{81}$ FG of $3 \%$ FGC

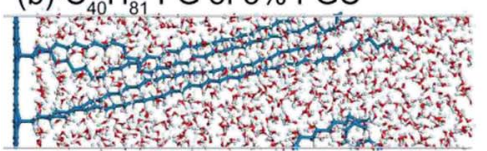

(c) $-\mathrm{C}_{40} \mathrm{H}_{81}$ FG of $4.5 \%$ FGC

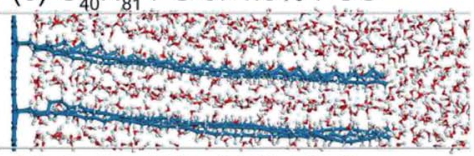

(d) $-\mathrm{C}_{40} \mathrm{H}_{81}$ FG of $7 \%$ FGC

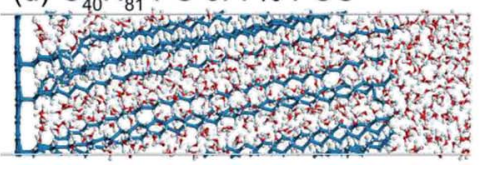

(e) $-\mathrm{C}_{90} \mathrm{H}_{181}$ FG of $1.5 \%$ FGC

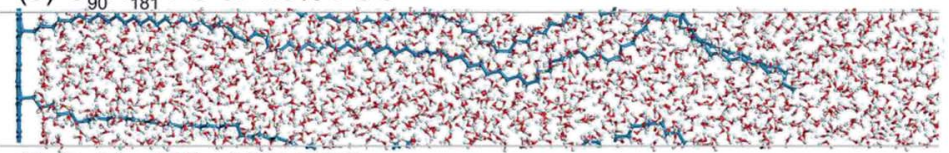

(f) $-\mathrm{C}_{90} \mathrm{H}_{181}$ FG of $3 \%$ FGC

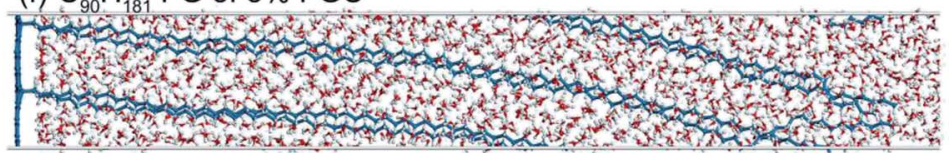

(g) $-\mathrm{C}_{90} \mathrm{H}_{181}$ FG of $4.5 \%$ FGC

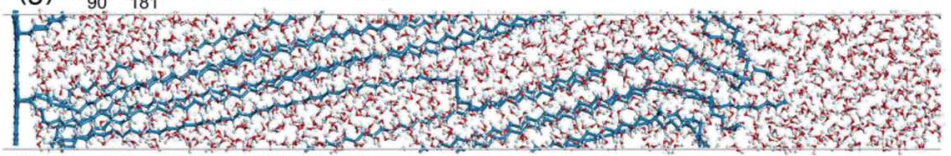

(h) $-\mathrm{C}_{90} \mathrm{H}_{181} \mathrm{FG}$ of $7 \%$ FGC

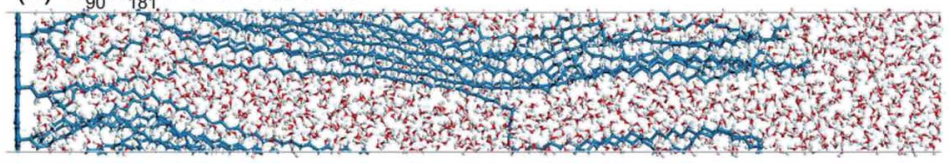

Fig. 6 Comparisons of different coverage in typical-length model schematic diagrams after NEMD simulations. The alkane chains gradually become straight in the water as the coverage increases in general in $-\mathrm{C}_{40} \mathrm{H}_{81} \mathrm{FGs}$. However, at $4.5 \% \mathrm{FGC}$, the ends of alkane chains start showing obvious bending and knots in $-\mathrm{C}_{90} \mathrm{H}_{181}$ FGs. 
interfacial thermal transport between water and graphene, the interfacial thermal conductance increases monotonically with chain length and coverage in general. This phenomenon is consistent with our results showing that the interfacial thermal conductance changed with chain length at $3 \%$ FGC. However, the growth of the alkane chains did not lead to further improvement at coverage of $1.5 \%, 4.5 \%$, and $7 \%$ (Fig. 3). At low coverage $(1.5 \%)$, thermal transport across the interface was dominated by water molecules interacting directly with carbon atoms in the graphene layer. The improvement in thermal conductance due to alkane functionalization was not significant. As a result, the effect of chain length was buried by noise (standard deviation). At a coverage of $3 \%$, the interfacial thermal conductance increased with chain length due to additional energy paths between alkane chains and water, as expected. If the coverage is $>3 \%$, the interfacial thermal conductance did not increase with increasing chain length. We inferred that the increase of coverage at long alkane chains could lead to cluttered (or even entangled) chains and, thus, could not further improve the interfacial thermal conductance. As shown in Fig. 6, the alkane chains became straighter in general as the coverage increased for $-\mathrm{C}_{40} \mathrm{H}_{81} \mathrm{FGs}$, and this change corresponded to the conductance trend shown in Fig. 3(b). However, the morphology of the $-\mathrm{C}_{90} \mathrm{H}_{181}$ FGs alkane did not change monotonically with coverage. Though chains continued to become more extended from 1.5\% FGC to $3 \%$ FGC, the spatial span decreased due to bending and knots as

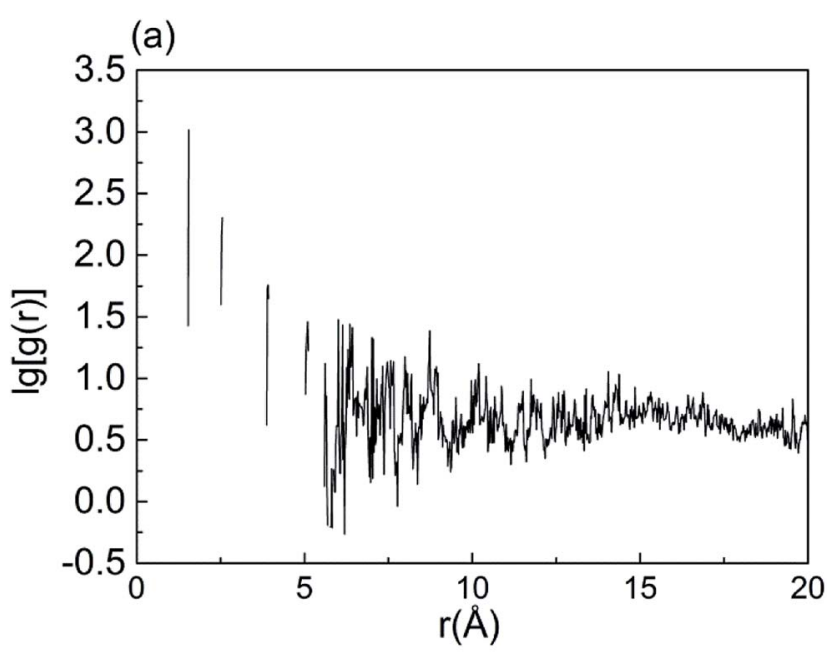

(b)

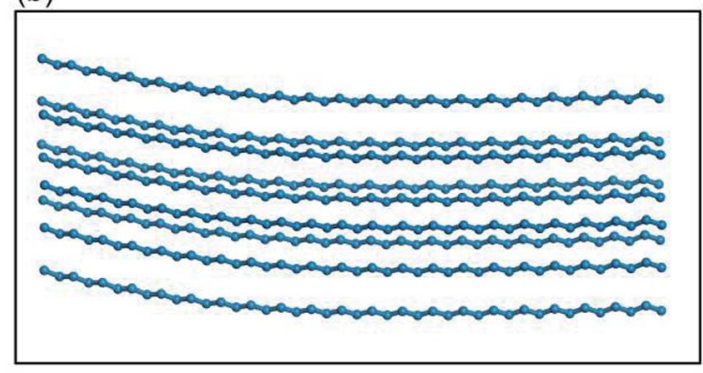

Fig. 7 (a) A typical radial distribution function (RDF) and (b) schematic diagram of $\mathrm{C}$-atom structure in undeformed $-\mathrm{C}_{90} \mathrm{H}_{181}$ chains of $7 \%$ FGC. the converge increased from $3 \%$ to $7 \%$ FGC. The $-\mathrm{C}_{90} \mathrm{H}_{181}$ alkane chains were also more aggregated compared with $-\mathrm{C}_{40} \mathrm{H}_{81}$ FGs, thereby hampering the entry of water molecules. These effects hindered thermal transport to a large extent, and explained the sudden decrease of $G / G_{0}$ s from $3 \%$ to $4.5 \%$ FGC (Fig. 3(b)).

Thermal transport in polymers is affected strongly by chain morphology ${ }^{47}$ To describe the structural changes, the radial distribution function (RDF) ${ }^{46,48-50}$ of carbon atoms in alkane chains was calculated to characterize the chain order. Sharper peaks in RDF calculations indicate a higher structure order. Fig. 7 shows that the RDF of carbon atoms of $-\mathrm{C}_{90} \mathrm{H}_{18}$ chains without bending and knots, and serves as a reference. As shown in Fig. 8, RDFs of $-\mathrm{C}_{90} \mathrm{H}_{181}$ FGs in $4.5 \%$ and $7 \%$ FGC had broader and smoother peaks compared with those of $1.5 \%$ FGC, indicating that the alkane chains become more chaotic when coverage increased. Compared with the RDFs of $-\mathrm{C}_{40} \mathrm{H}_{81} \mathrm{FGs}$, the overall peaks of $-\mathrm{C}_{90} \mathrm{H}_{181}$ FGs were gentler, indicating a higher level of disorder and lower thermal transport.
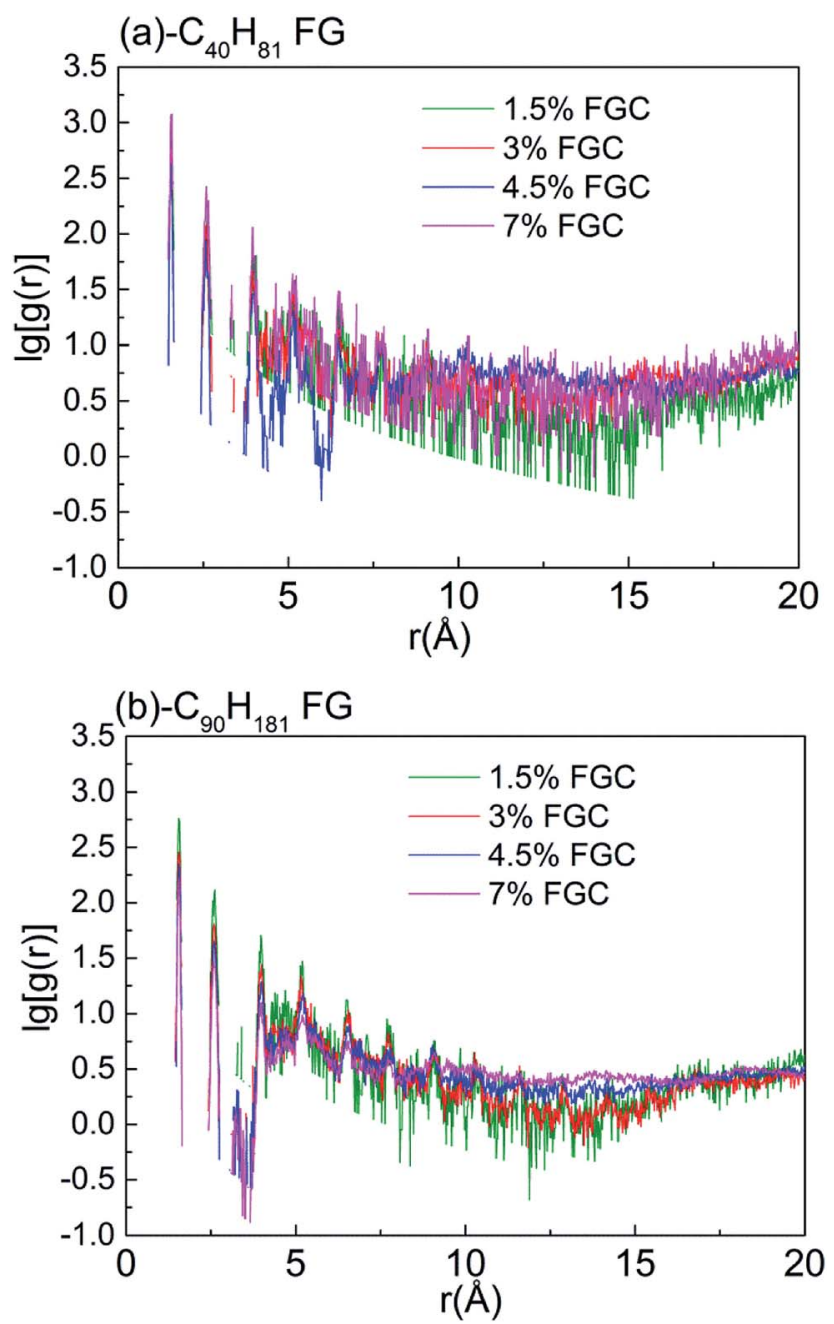

Fig. 8 Radial distribution functions (RDFs) of $\mathrm{C}$ atoms in (a) $-\mathrm{C}_{40} \mathrm{H}_{81}$ and (b) $-\mathrm{C}_{90} \mathrm{H}_{181}$ chains after NEMD simulations. The RDFs of $-\mathrm{C}_{90} \mathrm{H}_{181} \mathrm{FGs}$ in $4.5 \%$ and $7 \%$ FGCs have broader and smoother peaks compared with those of $1.5 \% \mathrm{FGC}$, and the overall peaks are more gentle at $-\mathrm{C}_{90} \mathrm{H}_{181}$ FGs compared with $-\mathrm{C}_{40} \mathrm{H}_{81}$ FGs. 


\section{Conclusions}

The interfacial thermal conductance between multilayer graphene and water was investigated via NEMD simulations. The alkane chains in FG could improve thermal transport across the graphene-water interface greatly. The most improved interfacial thermal conductance was up to $121.0 \pm 11.4 \mathrm{MW} \mathrm{m}^{-2} \mathrm{~K}^{-1}$. The length and coverage of alkane chains were the major factors contributing to enhancements in interfacial thermal conductance. With analysis on phonon scattering and diffusion effect, we attributed the improvement to the addition of hydrogen, which facilitated phonon transport between graphene and water. The interfacial conductance increases with alkane coverage in general, and it also increases with the alkane chain length when the functionalized group coverage is about $3 \%$. We provided a general method to promote the interfacial thermal conductance of graphene-water interfaces by "tuning" the morphology of graphene and alkane chains. By revealing the fundamental mechanism at the molecular level, our findings provide guidance for development of high-performance graphene-water interfacial thermal transport.

\section{Conflicts of interest}

The authors declare no competing financial interest.

\section{Acknowledgements}

We thank Professor Ruining Wang for the kind help with computations. This work was supported by National Key Research and Development Program (2017YFB0601803), National Natural Science Foundation of China (51606192, 51606190), Natural Science Foundation of Hebei Province (A2017201219), and CAS Pioneer Hundred Talents Program. Calculations were supported by the National Supercomputer Center in Guangzhou and the Institute of Engineering Thermophysics, CAS.

\section{References}

1 K. S. Novoselov, A. K. Geim, S. V. Morozov, D. Jiang, Y. Zhang, S. V. Dubonos, I. V. Grigorieva and A. A. Firsov, Science, 2004, 306, 666.

2 C. Lee, X. Wei, J. W. Kysar and J. Hone, Science, 2008, 321, 385-388.

3 A. A. Balandin, S. Ghosh, W. Bao, I. Calizo, D. Teweldebrhan, F. Miao and C. N. Lau, Nano Lett., 2008, 8, 902.

4 Z. Lin, Y. Liu, Y. Yao, O. J. Hildreth, Z. Li, K. Moon and C. Wong, J. Phys. Chem. C, 2011, 115, 7120-7125.

5 A. A. Balandin, Nat. Mater., 2011, 10, 569.

6 R. Mas-Ballesté, C. Gómez-Navarro, J. Gómez-Herrero and F. Zamora, Nanoscale, 2011, 3, 20-30.

7 J. Y. Kim and J. C. Grossman, Nano Lett., 2015, 15, 28302835.

8 N. Xiao, X. Dong, L. Song, D. Liu, Y. Tay, S. Wu, L. J. Li, Y. Zhao, T. Yu and H. Zhang, ACS Nano, 2011, 5, 2749-2755.
9 X. An, T. Simmons, R. Shah, C. Wolfe, K. M. Lewis, M. Washington, S. K. Nayak, S. Talapatra and S. Kar, Nano Lett., 2010, 10, 4295-4301.

10 D. G. Cahill, W. K. Ford, K. E. Goodson, G. D. Mahan, A. Majumdar, H. J. Maris, R. Merlin and S. R. Phillpot, J. Appl. Phys., 2003, 93, 793-818.

11 D. G. Cahill, P. V. Braun, G. Chen, D. R. Clarke, S. Fan, K. E. Goodson, P. Keblinski, W. P. King, G. D. Mahan and A. Majumdar, Appl. Phys. Rev., 2014, 1, 251-265.

12 Z. Y. Ong and E. Pop, Phys. Rev. B: Condens. Matter Mater. Phys., 2011, 84, 9226-9231.

13 S. Ghosh, I. Calizo, D. Teweldebrhan and E. P. Pokatilov, Appl. Phys. Lett., 2008, 92, 151911-151913.

14 T. Zhang, A. R. Gans-Forrest, E. Lee, X. Q. Zhang, C. Qu, Y. S. Pang, F. Sun and T. F. Luo, ACS Appl. Mater. Interfaces, 2016, 8, 33326-33334.

15 X. F. Wei, T. Zhang and T. F. Luo, ACS Energy Lett., 2017, 2, 2283-2292.

16 Y. Ma, Z. Zhang, J. Chen, K. Sääskilahti, S. Volz and C. Jie, Carbon, 2018, 135, 263-269.

17 D. Alexeev, J. Chen, J. H. Walther, K. P. Giapis, P. Angelikopoulos and P. Koumoutsakos, Nano Lett., 2015, 15, 5744-5749.

18 B. Y. Cao, J. H. Zou, G. J. Hu and G. X. Cao, Appl. Phys. Lett., 2018, 112, 041603.

19 Y. Wang, Z. Qin, M. J. Buehler and Z. P. Xu, Nat. Commun., 2016, 7, 12854.

20 D. Cai and M. Song, J. Mater. Chem., 2010, 20, 7906-7915.

21 H. Han, Y. Zhang, N. Wang, S. M. Kabiri, Y. Ni, Z. Y. Mijbil, E. Michael, S. Xiong, S. Kimmo and M. Murali, Nat. Commun., 2016, 7, 11281.

22 J. Yang, Y. Pang, W. Huang, S. K. Shaw, J. Schiffbauer, M. A. Pillers, X. Mu, S. Luo, T. Zhang and Y. Huang, ACS Nano, 2017, 11, 5510.

23 C. Teng, C. M. Ma, K. Chiou and T. Lee, presented in part at the 2010 5th International Microsystems Packaging Assembly and Circuits Technology Conference, 20-22 Oct. 2010, 2010.

24 Y. Wang, H. F. Zhan, Y. Xiang, C. Yang, C. M. Wang and Y. Y. Zhang, J. Phys. Chem. C, 2015, 119, 12731-12738.

25 M. Wang, N. Hu, L. Zhou and C. Yan, Carbon, 2015, 85, 414421.

26 H. Tang, H. Ye, X. Chen, X. Fan and G. Zhang, presented in part at the 2017 18th International Conference on Thermal, Mechanical and Multi-Physics Simulation and Experiments in Microelectronics and Microsystems (EuroSimE), 3-5 April 2017, 2017.

27 S. Lin and M. J. Buehler, Nanotechnology, 2013, 24, 165702. 28 X. Wei, Z. Teng and T. Luo, ACS Appl. Mater. Interfaces, 2017, 9, 33740-33748.

29 B. Radha, A. Esfandiar, F. C. Wang, A. P. Rooney, K. Gopinadhan, A. Keerthi, A. Mishchenko, A. Janardanan, P. Blake and L. Fumagalli, Nature, 2016, 538, 222-225.

30 H. Han, S. Merabia and F. Müllerplathe, J. Phys. Chem. Lett., 2017, 8, 1946-1951.

31 E. Sadeghinezhad, M. Mehrali, R. Saidur, M. Mehrali, S. T. Latibari, A. R. Akhiani and H. S. C. Metselaar, Energy Convers. Manage., 2016, 111, 466-487. 
32 P. A. Denis and F. Iribarne, Chem.-Eur. J., 2012, 18, 75687574.

33 F. Müllerplathe, J. Chem. Phys., 1997, 106, 6082-6085.

34 P. C. Howell, J. Comput. Theor. Nanosci., 2011, 8, 2129-2143.

35 W. H. Press, B. P. Flannery, S. A. Teukolsky and W. T. Vetterling, Numerical recipes: the art of scientific computing, Cambridge University Press, Cambridge, 1986.

36 S. L. Mayo, B. D. Olafson and W. A. Goddard, J. Phys. Chem., 1990, 94, 8897-8909.

37 P. B. Allen, J. L. Feldman, J. Fabian and F. Wooten, Philos. Mag. B, 1999, 79, 1715-1731.

38 E. T. Swartz and R. O. Pohl, Rev. Mod. Phys., 1989, 61, 605668.

39 J. Moon, B. Latour and A. J. Minnich, Phys. Rev. B, 2018, 97, 024201.

40 Q. Liang, X. Yao, W. Wang, Y. Liu and C. P. Wong, ACS Nano, 2011, 5, 2392-2401.

41 Y. Zhou, Z. Fan, G. Qin, J. Y. Yang, T. Ouyang and M. Hu, ACS Omega, 2017, 3, 3278-3284.
42 M. D. Losego, M. E. Grady, N. R. Sottos, D. G. Cahill and P. V. Braun, Nat. Mater., 2012, 11, 502-506.

43 P. E. Hopkins, M. Baraket, E. V. Barnat, T. E. Beechem, S. P. Kearney, J. C. Duda, J. T. Robinson and S. G. Walton, Nano Lett., 2012, 12, 590-595.

44 J. M. Dickey and A. Paskin, Phys. Rev., 1969, 188, 1407-1418. 45 M. R. Lee and D. Ben-Amotz, J. Chem. Phys., 1993, 99, 1007410077.

46 J. M. Larkin and A. J. H. Mcgaughey, Phys. Rev. B: Condens. Matter Mater. Phys., 2014, 9, 620-637.

47 X. Wei, T. Zhang and T. Luo, Phys. Chem. Chem. Phys., 2016, 18, 32146.

48 T. Zhang and T. Luo, J. Appl. Phys., 2012, 112, 1571.

49 E. Lorch, J. Phys. C: Solid State Phys., 2002, 2, 229.

50 K. Laaziri, S. Kycia, S. Roorda, M. Chicoine, J. L. Robertson, J. Wang and S. C. Moss, Phys. Rev. B: Condens. Matter Mater. Phys., 1999, 60, 13520-13533. 